\title{
Special content of this issue
}

\author{
Alexander Steinbüchel
}

Published online: 1 February 2011

(C) Springer-Verlag 2011

With this editorial, I would like to draw your attention to seven manuscripts whose contents were presented at the "International Symposium on Biopolymers 2010" (ISBP 2010). This biennial conference was held from October 3 to 7, 2010, in Stuttgart (Germany) and has been organized by Dieter Jendrossek and the DECHEMA. This conference has now been held already 12 times. In total, 96 oral presentations and 90 posters were presented during the 4 days of the conference.

The seven manuscripts are just a selection of contributions focusing on polyesters of the type of polyhydroxyalkanoates (PHA) on one side and on microbiological and/or biotechno- logical aspects on the other side. A mini review summarizes the current state on the production of PHAs from palm oil (Sudesh et al.). The other six publications are original papers and report on the use of palm oil for growth and PHA production by Ralstonia eutropha (Budde et al.), on a comparison of promoters and vectors suitable for gene expression in $R$. eutropha (Fukui et al.), on novel short carbon-chain-length constituents of PHAs (Lau et al.), on functionalized medium carbon-chain-length constituents of PHAs (Escapa et al.), on an unusual PHA depolymerase (Sznajder \& Jendrossek), and finally on the use of PHA nanoparticles for the release of cancer cell inhibitors ( $\mathrm{Lu}$ et al.).

A. Steinbüchel $(\square)$

Institute for Molecular Microbiology and Biotechnology,

University of Münster,

Corrensstraße 3,

48149 Münster, Germany

e-mail: steinbu@uni-muenster.de 